\title{
Screening commercial entomopathogenic fungi for the management of Diaphorina citri populations in the Lower Rio Grande Valley, Texas, USA
}

\author{
Justin Wendel · Jonathan Cisneros $\cdot$ Stefan Jaronski $\cdot$ Christopher Vitek • \\ Matthew Ciomperlik · Daniel Flores $(\mathbb{D}$
}

Received: 1 July 2021 / Accepted: 23 December 2021 / Published online: 22 January 2022

This is a U.S. government work and not under copyright protection in the U.S.; foreign copyright protection may apply 2022 , corrected publication 2022

\begin{abstract}
Ten strains of entomopathogenic ascomycete fungi, sourced from commercial formulations of blastopore or conidiospore formulations, were tested in 14 different formulations in a primary acquisition/direct spray bioassay against adult Asian citrus psyllid (Diaphorina citri Kuwayama (Hemiptera: Liviidae)). The Cordyceps (Isaria) javanica Apopka 97-C (conidia) strain was used as the standard. A statistical ranking system was established in which top performing pathogenic strains were selected for further screening and eventual field trials. Modified Potter-type spray towers were utilized to deliver a range of doses of viable spores to adult $D$. citri in an aqueous spray consistent with the rate of spores per
\end{abstract}

Handling Editor: Everton Kort Kamp Fernandes

Fernandes.

J. Wendel · C. Vitek

University of Texas Rio Grande Valley, Edinburg, TX, USA

J. Cisneros · M. Ciomperlik · D. Flores $(\bowtie)$ USDA APHIS PPQ S\&T Insect Management \& Molecular Diagnostics Laboratory, Edinburg, TX, USA

e-mail: daniel.flores@usda.gov

S. Jaronski

USDA ARS NPARL, Sidney, MT, USA

Present Address:

S. Jaronski

Jaronski Mycological Consulting LLC, Blacksburg, VA, USA hectare often used in real-world spray applications. Mortality was assessed after a seven-day incubation period under controlled climate conditions reflecting those in the Lower Rio Grande Valley (LRGV) of Texas, USA. Of the 14 preparations, the strains Metarhizium anisopliae E9, C. fumosorosea Ifr9901, Beauveria bassiana ATCC 74040 and ANT-03, M. anisopliae ESALQ1037, and M. robertsii DWR2009, showed greater levels of mortality than the standard, Apopka 97-C, in the laboratory setting. Of those six, two (Ifr9901 and ANT-03) were selected for further evaluation based on efficacy, commercial availability, geographical registration, and market outlook on production.

Keywords Mycoinsecticides - Entomopathogenic fungi - Diaphorina citri - Asian citrus psyllid · Hemiptera $\cdot$ Liviidae

\section{Introduction}

The Asian citrus psyllid (ACP), Diaphorina citri Kuwayama (Hemiptera: Liviidae) is a well known citrus pest. Having first been identified on the east coast of Florida, USA in 1998, and later, in 2008, in southern California, USA, it has since been found across the southern USA citrus growing regions (Bayles et al. 2017). The D. citri is well known for facilitating the spread of Candidatus Liberibacter spp., the causative agent of citrus greening disease, also known 
as Huanglongbing (HLB) (Grafton-Cardwell et al. 2013; Hall et al. 2013; Pelz-Stelinski et al. 2010).

The bacterium resides in the phloem of citrus trees and is acquired by the phloem feeding D. citri (Bové 2006). The bacterium colonizes in the gut of the $D$. citri where it is later transferred during feeding events to other non-infected trees, thus spreading the disease (Kruse et al. 2017). Due to this method of transmission and overall nature of the disease, citrus greening presents a unique problem. Where most crop pests tend to produce physical damage to plants reducing longevity or production with sheer population numbers and physical damage to the crop, it only takes a single infected psyllid to spread this progressive and degenerative disease to multiple plants.

Diaphorina citri has only emerged as a pest insect in the USA in the last decade and more immediate tools such as synthetic insecticides were initially sought out to overcome the impending panzootic, especially in commercial citrus (Grafton-Cardwell 2006). More recently, however, attention has turned to smaller, less managed citrus groves and residential citrus (Sétamou et al. 2020; Chow et al. 2019). This has created a niche demographic of individuals who would prefer not to use synthetic insecticide control methods and keep their citrus trees and residences organic, or at the least free of pesticide residues.

Several alternative means of management have been investigated, one of which is biocontrol using a natural parasitoid of the insect. This biocontrol tool, which became a mainstay in an IPM strategy against D. citri, uses the natural predator/ectoparasitoid Tamarixia radiata (Flores and Ciomperlik 2017). A rearing and release program at the USDA APHIS PPQ S\&T Insect Management \& Molecular Diagnostics Laboratory (IMMDL) saw a $91.2 \%$ decline in psyllid populations between the years 2010 and 2016 after releases were made in residential areas (Flores and Ciomperlik 2017).

Insect pathogenic fungi, particularly the Ascomycete Hypocreales genera Beauveria, Cordyceps (Isaria), and Metarhizium, and to a lesser extent, Akanthomyces (Lecanicillium), have been developed worldwide as biocontrol agents of a wide range of insects and other arthropods. As mycoinsecticides they possess the unique quality of being used for both foliar spray and soil applications and remain an organic means of pest control. In the past, mycoinsecticides have been successful in the population management of such pests as the sweet potato whitefly, Bemisia tabaci (Gennadius), Colorado potato beetle, a variety of aphids, and many other pest species (Campbell et al. 1985; Faria and Wraight 2001; Klinger et al. 2006; Spence et al. 2019).

Strains of several entomopathogenic fungi are commercially available in the USA. Several of these strains were commercialized during the time of the sweet potato whitefly outbreak in the 1990s (Osborne and Landa 1992; Wraight et al. 1998), although they are registered for a range of insects. Exposure of D. citri nymphs to entomopathogenic fungi has been noted to decrease egg deposition from the ectoparasitoid Tamarixia radiata, presumably to prevent larval emergence in a terminally infected host (Chow et al. 2018). This observation, as well as those made in other studies, implies an ability of parasitic and predatory insects to identify hosts or prey which have been infected, limiting the casualty rate among the parasites/predator's population (Meyling and Pell 2006; Tasin et al. 2012). Overall, entomopathogenic fungi show minimal threat to the predatory and parasitic insects that prey upon their hosts (Hamdi et al. 2011; Kapongo et al. 2008; Al Mazra' awi et al. 2006; Roy and Pell 2000).

While several commercial mycoinsecticides are available, only $C$. javanica strain Apopka 97, as PFR97®, has been labeled for use against Asian citrus psyllids. The goal of this study was to screen all the registered commercial fungi currently available in the USA for efficacy against $D$. citri using a set of standardized protocols to determine if any were as or more efficacious than Apopka 97-C, and thus capable of assisting current IPM strategies in the management of $D$. citri populations. The screening process was designed to reduce the final candidates to a reasonable number of strains for future field testing. The development of this protocol was driven by the need for a standardized method of determining mortality that reflected spore deposition rates observed in field applications.

\section{Materials and methods}

Insects

Diaphorina citri used for the bioassays were obtained from the USDA IMMDL colony in Edinburg, TX, USA. Insects were reared on orange jasmine (Murraya paniculata) in accordance with the protocol described in Flores and Ciomperlik (2017). An 
average of 35-40 adult ACP was collected from cages using a manual aspirator (BioQuip, Model 1135C), and stored at $4{ }^{\circ} \mathrm{C}$ for at least $2 \mathrm{~h}$ to acclimate before initiating sprays. Vials were then placed in a $-15^{\circ} \mathrm{C}$ freezer for 3 min before being processed under primary acquisition (direct spray) protocols. The duration of each spray procedure takes an average of $30 \mathrm{~s}$, therefore vials of psyllids were placed into the freezer every $30 \mathrm{~s}$ to maintain a steady supply of cold-anesthetized insects. This time and temperature regime was empirically determined to produce adult ACP that were sufficiently cold stunned to process through the spray protocol and minimize cold-treatment induced mortality.

\section{Fungi}

Samples of the commercial mycoinsecticides (Table 1) were obtained from either the manufacturers or commercial sources, except for Beauveria bassiana strain GHA, which was obtained as a technical grade conidial powder. The fungi were isolated from the formulations as single colonies and subsequently subcultured. Technical grade conidial powders for each strain were produced via solid substrate fermentation using methods as outlined in Jaronski and Jackson (2012). Conidia were dried to a water activity of $<0.3 \mathrm{Aw}$ and stored at $4-7{ }^{\circ} \mathrm{C}$ until use. In addition, several of the mycoinsecticide products that were in the form of dried blastopore preparations $(C$. javanica Apopka 97, and C. fumosorosea PPRI5539) were tested as such in addition to their conidial form.

\section{Bioassays}

For each bioassay a stock solution of approximately $1.0 \times 10^{9} \mathrm{ml}^{-1}$ was suspended in $0.1 \%$ Tween 80 using a glass Tenbroeck homogenizer. From this initial suspension a 100-fold dilution was then counted on a standard hemocytometer to determine spore concentration of the stock. An aliquot from the 100-fold dilution was also plated on potato dextrose agar (BD Scientific; Cat\# 254920) for $16-18 \mathrm{~h}$ at $27^{\circ} \mathrm{C}$ and percent viability of the spores was determined. This was performed by staining an excised block of agar with cotton blue stain (MycoPerm; Fisher Cat \# 23769320) and determining viable and non-viable spores with 400X bright field microscopy. At 16-18 h, any spore that had a germ tube of any length was considered viable, while any spore without a germ tube, stained or unstained was considered non-viable.

From these data, the concentrations of the stock suspensions, in terms of viable spores $\mathrm{ml}^{-1}$ were determined and utilized to make all further serial dilutions for the bioassay, creating a normalized level of comparison between each strain. A broad range of doses, including $3.0 \times 10^{6}, 1.0 \times 10^{7}, 3.0 \times 10^{7}, 1.0 \times$ $10^{8}$, and $3.0 \times 10^{8}$ viable spores $\mathrm{ml}^{-1}$ was employed. A spray of $0.4 \mathrm{ml}$ of each dose reflected a range of spore deposition rate comparable to field sprays based on the recommended use rates of the existing commercial mycoinsecticides. The rates were confirmed using spores $\mathrm{mm}^{-2}$ derived from replicate counts of spores deposited on an agar block, which had been placed in the spray chamber, and extrapolating the data to spores $\mathrm{ha}^{-1}$.

\section{Potter spray tower}

The fungal spores were applied using a series of Potter-type spray towers constructed from acrylic tanks ( $\mathrm{D}=30 \mathrm{~cm}, \mathrm{H}=40 \mathrm{~cm}$ ) fitted with PVC endcaps. A series of polyethylene blocks were mounted, one atop the other, to the lid, with a $1 \mathrm{~cm}$ wide, $90^{\circ}$ angle cut on each of the touching faces to allow the insertion and adjustable placement of a three-prong adjustable chemistry clamp. A $2.5 \mathrm{~cm}$ hole was cut in the center of the lid, and an airbrush (Paasche®, Model H0417) was mounted in the clamp and positioned directly above the hole. This airbrush was connected to an air compressor (Dewalt; Model D55140), fitted with an air tank reservoir to allow smooth consistent airflow. Regulators were added to further control air pressure and airflow, and in-line moisture traps were added to prevent rust within and prolong the life of the airbrushes. The base of the tower was raised $2.5 \mathrm{~cm}$ using polyethylene blocks $(\mathrm{H}=2.5 \mathrm{~cm}, \mathrm{~W}=5 \mathrm{~cm}$, $\mathrm{L}=5 \mathrm{~cm}$ ), and an $8 \mathrm{~cm}$ hole was cut into the base of the tower to allow for transient airflow. A Whatman No. 1 filter paper was taped over the hole to prevent spores from leaving the tower without restricting airflow. The lower portion of a test tube rack (Globe Scientific, Model 456930) was employed to raise the samples slightly and allow clean passage of air around the samples and through the exhaust port as designed. The top, main chamber, and base of the towers were marked to ensure the alignment of the attached brush and base was consistent. Calibration 


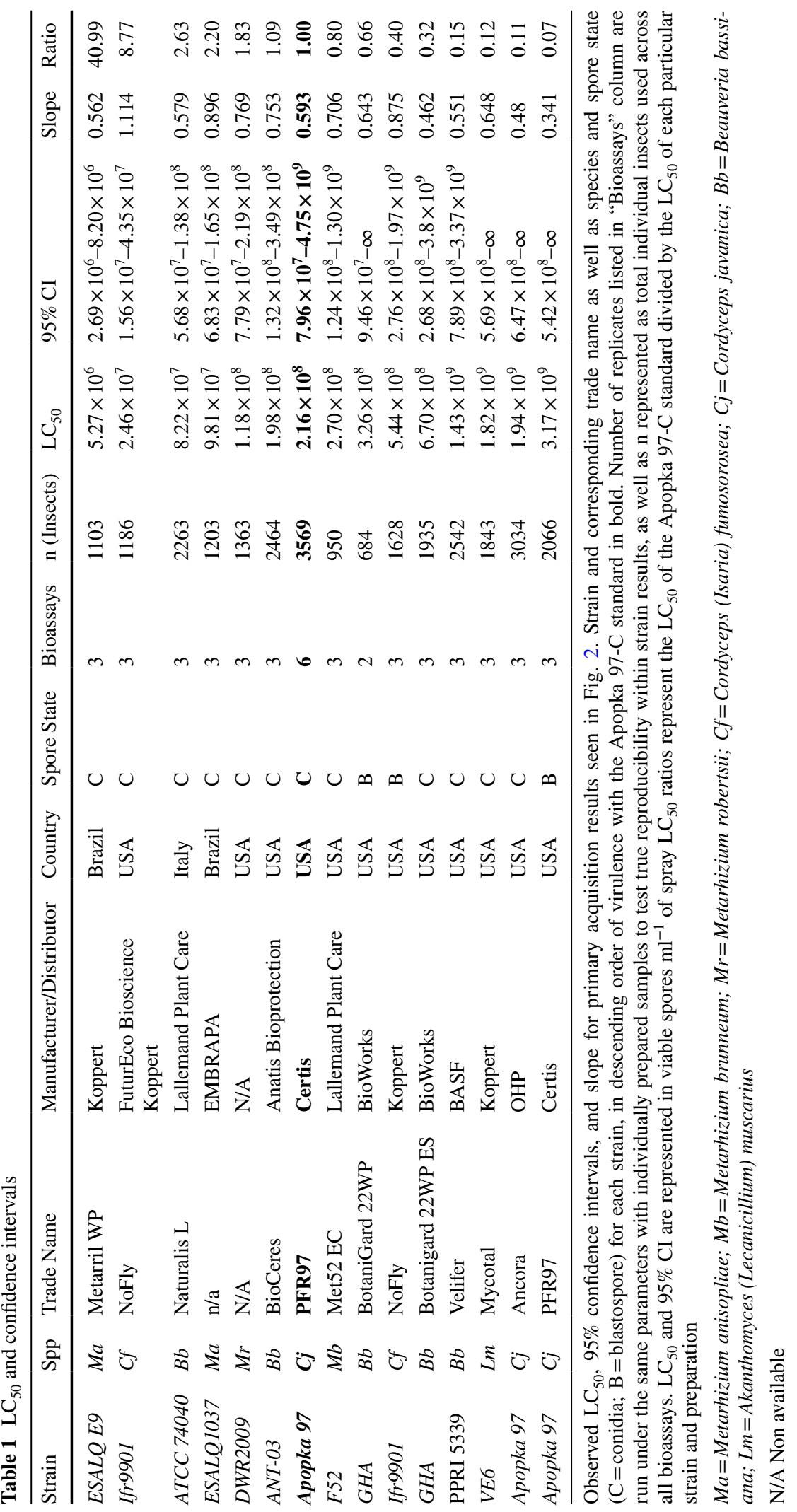


of towers was performed prior to each spray sequence to ensure the samples were placed in the center of the spore dispersion pattern for each spray. This process produced consistent deposition of spores on samples, avoiding the lower density outer edges of the spray.

Spray pattern tests were performed using water sensitive spray cards (Syngenta) to achieve optimal spore deposition/density, and to ensure testing parameters were representative of what would be seen in future field trials. Water sensitive spray cards were cut into $1 \mathrm{~cm}$ squares and placed within the spray area in the tower to determine spore deposition/density via droplet dispersion.

Sprays were conducted at $70,100,140$, and $170 \mathrm{kPa}$. Spray cards were photographed and analyzed using ImageJ (Schneider et al. 2012) for percent coverage, droplet size, and consistency of deposition. Based on the results of this, all reported testing was conducted at $140 \mathrm{kPa}$.

\section{Spore deposition}

Routine determination of spore deposition during the bioassays was not conducted due to workload constraints. However, spore deposition was performed in a parallel study to draw correlations between the quantity of spores deposited per $\mathrm{cm}^{2}$ during bioassays versus actual field applications in a grove setting. According to Wraight and Carruthers (1999) the best rates of pest control are seen in field applications of $10^{13}-10^{14}$ spores $\mathrm{ha}^{-1}$. The dose rates which were selected accurately reflect these field application ranges and show little variation at each dose between strains.

\section{Post-treatment handling of insects}

Flush (orange jasmine cuttings) enclosures were constructed from $4 \mathrm{~cm}$ diameter plastic fluorescent tube guards and the corresponding end caps were modified for use. Tubes were trimmed to $30 \mathrm{~cm}$ length. End caps were manufactured with a $2 \mathrm{~cm}$ diameter hole with organza mesh glued to the inside of the caps to ensure appropriate ventilation as well as containment of $D$. citri. Florist aqua-tubes were filled with a $0.30 \%$ hydroponic solution (General Hydroponics®; Maxi$\mathrm{Gro}^{\mathrm{TM}}$ ) to maintain citrus flush viability throughout the seven-day incubation period, during which adult ACP were expected to feed on the flush. Laboratory film was wrapped around the cap of the water tube and the entry point of the flush to ensure minimal evaporation of solution and to prevent the undesirable mortality of $D$. citri that might make their way into the solution. Orange jasmine flush viability was best served by utilizing flush $20 \mathrm{~cm}$ tall, with an average of 20-25 leaves, and slightly observable woody secondary growth on the lower stem. Racks for finished flush tubes were retrofitted with screen bottoms to ensure continuous airflow and control of internal temperature and humidity. Flush that had desiccated during the incubation period was omitted from mortality analysis because a lack of viable food source was presumed to influence final mortality data.

At $24 \mathrm{~h}$ post-treatment, deceased psyllids were removed from the specimen tubes as deaths in this time period were due to factors unrelated to the tested fungal pathogens. Remaining live psyllids were left for the full seven-day period. Upon counting of live/ dead insects on day 7, those replicate groups that had a control with a mortality greater than $10 \%$ were completely omitted from the study.

Environmental parameters

Post-treatment, the flush with psyllids was incubated at $26^{\circ} \mathrm{C}$ and $80 \% \mathrm{RH}$, with a L:D 12:12 photoperiod, using Percival ${ }^{\circledR}$ incubators. These parameters were chosen to reflect averages during 2016, as recorded at the USDA IMMDL via remote weather monitoring station.

\section{Screening protocol}

In order to elucidate the best possible candidates for future field trials and lower the number of tested strains, an approach was developed that would first narrow the number of candidate strains to those deemed most effective against adult $D$. citri using a direct spray, "primary acquisition" method. The efficacy of each of the strains was determined relative to a standard mycoinsecticide, $C$. javanica strain Apopka 97-C. This primary screening used 35-40 adult $D$. citri, were sprayed with one of the five selected doses and the bioassay replicated at least three times.

Once inoculated, the insects were placed in flush tubes with healthy flush and incubated for seven days. Upon the 7 th day, flush tubes were removed from 
incubators and the psyllids were counted to determine dead and live insects, and any notes pertaining to flush health, observed mycosis, or other anomalies (loss of hydroponic solution, presence of parasites/ predators, presence of saprophytic fungi, etc.) were recorded.

\section{Statistical analysis}

The assignment of $D$. citri and orange jasmine flush to the treatments was randomized throughout the experiment. Fungal strains were tested in replicates of three flush for each dose and the untreated control (carrier only). A C. javanica Apopka 97-C was included as a treated control along with other sprays as a standard. Dead and live psyllids were counted in each tube after seven days and totals were tracked through the entirety of the screening process. Probit analysis was performed using POLO statistical analysis software (LeOra Software Inc.)

Significance between strains was determined using the probit calculated $95 \%$ confidence interval overlap as seen in Gange et al. (2019) as well as Khashaveh and Chelav (2013). Confidence intervals between a strain and Apopka 97-C standard that overlapped were considered non-significant while those that did not overlap were considered significantly different.

As the method of application used in this study offers insight into real-world application efficacy, it also inherently produces increased variation within bioassays. Assays selected for statistical analysis had to pass the low heterogeneity standards of the statistical software POLO Plus (LeOra). Those with a higher heterogeneity were omitted from probit analysis but are depicted here in a cumulative box and whisker graph (Fig. 1) to observe the overall dose response despite variation and outliers.

\section{Results}

$\mathrm{LC}_{50}$

Metarhizium anisopliae E9 conidia had the lowest $\mathrm{LC}_{50}$ (Table 1) of the fungi tested by a significant margin. Ifr9901 conidia ranked second among those tested. These two strains were the only two strains to show increases in efficacy which was statistically significant compared to the standard Apopka 97-C (as noted by the lack of overlap in confidence intervals between those strains and the standard; Litchfield and Wilcoxon 1949). The remaining strains with $\mathrm{LC}_{50}$ values lower than Apopka 97-C still remain on the lower end of the confidence interval overlap, which may also be due in part to the wide breadth of Apopka 97-C confidence interval. Ratios in Table 1 are relative to the standard and show fold change in efficacy (values $>1$ show increased efficacy while values $<1$ show decrease in efficacy).

\section{Percent mortality}

The trendlines lines as observed in Fig. 2 show comparable overall dose responses between those bioassays which were analyzed through probit analysis.

\section{Discussion}

This study is one of the few that concentrates on all or most of the available commercial mycoinsecticides currently registered in the USA. In most published studies evaluating fungi against $D$. citri, or even other insects, the tendency has been to ignore registered strains in favor of the authors' favored experimental strains. While this perspective does contribute valuable information about strain specificity as well as general efficacy of entomopathogenic fungi, the practical aspects of registration and commercialization of the new strains, however, are too often ignored. The regulatory requirements of many countries pose considerable financial barriers to implementation (commercialization) of new strains and therefore supports the idea of screening currently registered products.

The Apopka 97 strain was selected as a standard (in its conidial form) by which each other product strain mortality would be tested. Existing data regarding Apopka 97 for ACP was a factor in this decision, as research was either limited or non-existent with the other strains at the time of the onset of this project (Avery et al. 2011; Kumar et al. 2017). Under the parameters of our screening protocols, those strains that presented with an efficacy and virulence greater than that of Apopka 97 would be selected for further screening, while those that proved less virulent and efficacious would be omitted. 

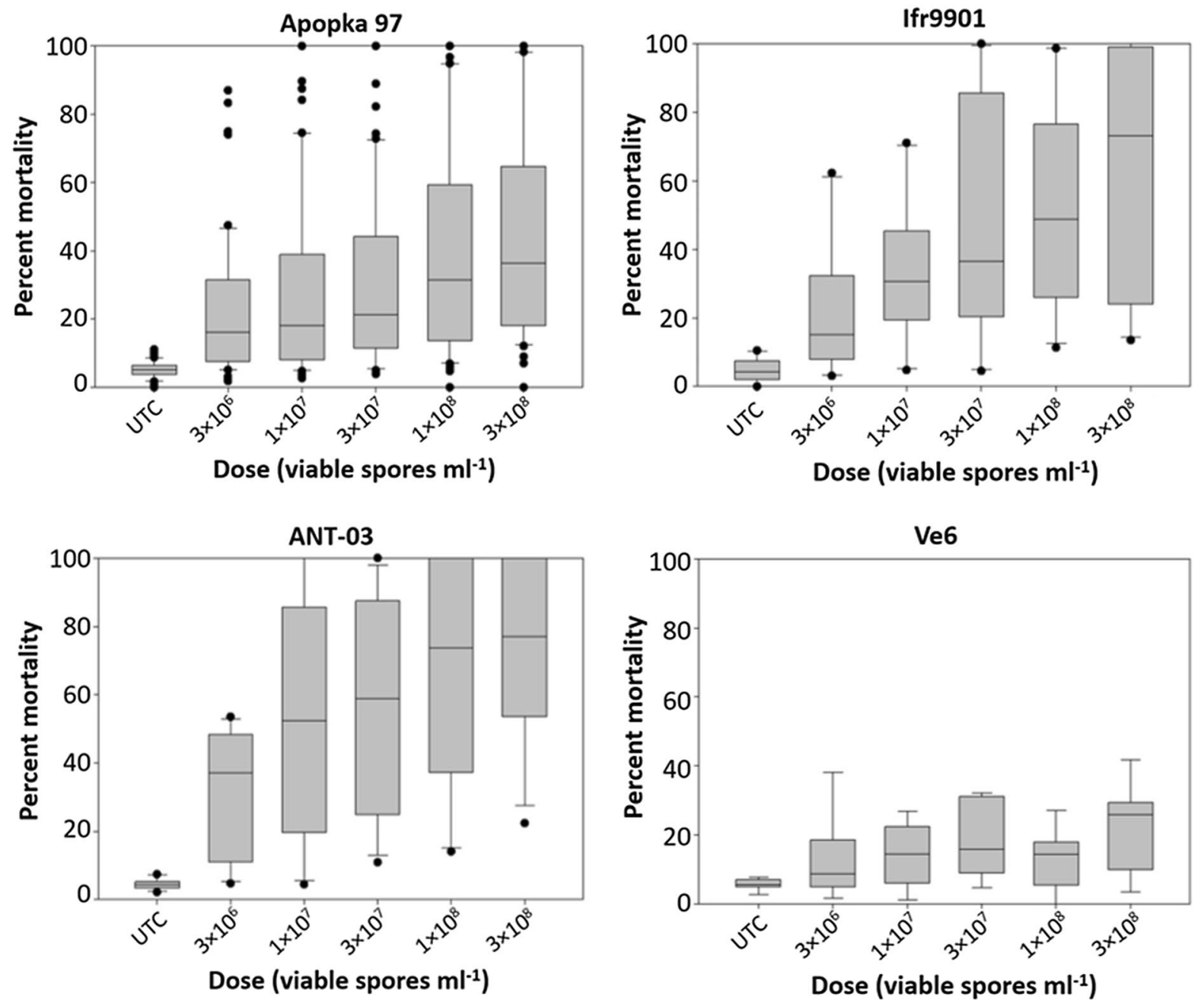

Fig. 1 Box and whisker representation of raw data. The area from lower whisker to lower box depicts first quartile of data, lower box depicts 2 nd quartile, upper box represents 3rd quartile, and area from upper box to upper whisker represents 4 th quartile. Dots along the plot indicate outliers. Probit analysis of bioassays data required utilizing datasets with a low enough

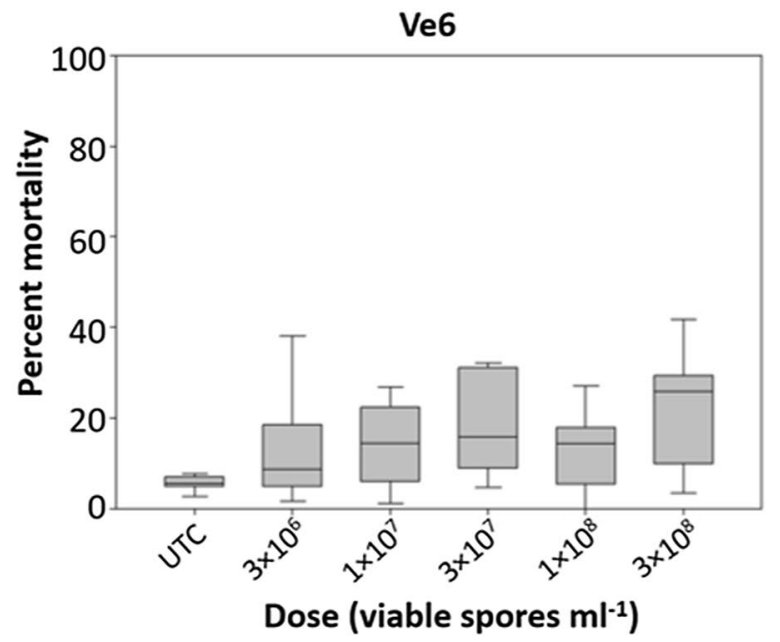

heterogeneity that statistical computation could be achieved. Well defined trends in the dose response are observed in top performing strains (Ifr9901, ANT-03), while low end performers show little no discernable dose response (Ve6). Apopka 97-C shown as standard

Our work represents an effort to identify a viable, field-ready tool to utilize as part of an integrated pest management plan to reduce and manage the population of ACP in the Lower Rio Grande Valley of Texas, USA. By screening commercialized mycoinsecticides for the most efficacious, we build the rationale for immediate implementation, and for those mycoinsecticides which are not registered in the USA, the rationale for an expedited registration. Pest management in this specific field requires vector control to mitigate the spread of the bacterial

disease until efforts to treat diseased trees are fruitful. The spread of citrus greening may be slowed by lowering the population of $D$. citri in residential areas and in conjunction with other pest management protocols. While this is essentially a stopgap measure and not a treatment for the disease, it is a viable tactic which grants a window of opportunity for those exploratory studies to determine an effective treatment.

The final field trial analysis may reveal realistic and practical trends by using spore concentrations 


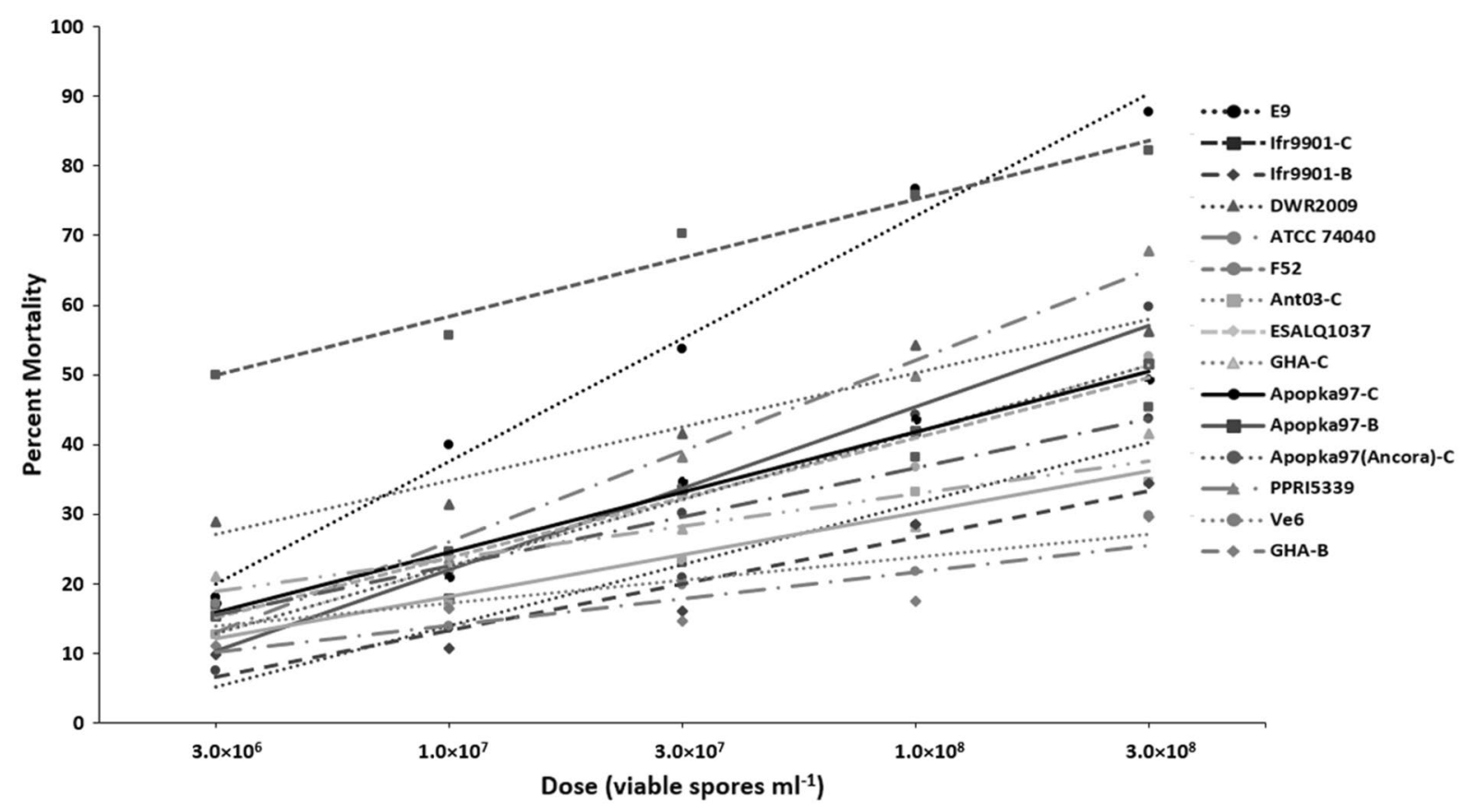

Fig. 2 Representative strain virulence under primary acquisition of all candidate strains. Average percent mortality across all strains for each dose, with the standard (Apopka 97-C) denoted by black solid line. Trendlines represent predicted mortality for respective strain at the relative dose. Legend is

and dose volumes that matched the range of recommended spores per acre in crop application. Methodology for administering spores varies widely, from whole plant and insect immersion, to individual droplet deposition. These methods are determined by the overall hypothesis of the individual study as well as size of the target insect. This study's aim was to closely mimic the type of primary acquisition that would be observed in field application, while maintaining tight control and replication within the laboratory setting.

Following the criteria of $\mathrm{LC}_{50}$ and efficacy, the top performing candidates relative to the standard were selected to undergo secondary acquisition screening, that is efficacy when the fungus spores are acquired by the insect from treated foliage as the psyllids move around. These fungi included, E9, Ifr9901-C, ATCC 74040, ESALQ1037, DWR2009, and ANT-03. However, E9 and ESALQ1037 strains are part of a previously commercialized product in Brazil known as Metarril, but not yet registered for use or sale in the USA. DWR2009 is a $M$. robertsii isolate from the listed in decreasing order of virulence based on the highest dose screened $\left(3.0 \times 10^{8}\right)$. While not conclusive, this figure is representative of the dose response of each respective strain. Letter " $C$ " or "B" following a strain denotes conidia or blastospore suspension respectively

ARSEF library which has no commercial counterpart but was included because it was the lead candidate in a USDA ARS mycoinsecticide development program (Jaronski, unpublished). These strains show promising results and incentive for manufacturers to pursue registration of these products within USA boundaries. However this is outside the scope of this study. Finally, ATCC 74040 (Naturalis) was obtained subsequent to the main screening program after the previous strains had been screened and selected for further testing (currently being conducted). As such ATCC 74040 was not among those selected to move forward. However it is currently undergoing screening through the aforementioned protocol. While the objective was to evaluate existing products readily available to residential and commercial growers, other factors such as availability, ease of commercial production (interpreted as lower cost to the consumer), and regional registration were all factors in the final progression to secondary acquisition. The omission of the aforementioned strains does not indicate a lack of potential in D. citri population management. However the 
support for registering a new product (in the case of E9, ESALQ1037, and DWR2009) is outside the scope of this current study. Nonetheless future work may include these alternatives.

Determination of spores $\mathrm{ha}^{-1}$ derived from the spores $\mathrm{mm}^{-2}$ observed in the bioassays was important to extrapolate the $\mathrm{LC}_{50}$ data to a quantity relevant to field application rates (Wraight et al. 1998, 2000, 2010). Too many studies report bioassay data merely in spores $\mathrm{ml}^{-1}$, which do not represent actual coverage even when the volume of spray per hectare is constant. A common published rate in single dose bioassays is $1.0 \times 10^{7}$ spores (conidia or blastospores) $\mathrm{ml}^{-1}$. The $\mathrm{LC}_{50}$ for Ifr9901-C and ANT-03 strains extrapolate to approximately $9.0 \times 10^{12}$ and $5.0 \times 10^{13}$ spores $\mathrm{ha}^{-1}$, respectively. Our extrapolation does not consider leaf area index of the target crop and assumes broadcast spray application as opposed to a targeted application to part of the plant canopy. A leaf area index for 5-year-old citrus (Citrus limon L., cv. Villafranca) in one study was calculated to be $2.8( \pm 4$, SD) (Cohen et al. 1997). In another study involving a commercial orchard of 'Valencia' sweet orange (Citrus sinensis L.), the leaf area index ranged from 4.9 to 6.0 (Morgan et al. 2014). As such, the spores $\mathrm{ha}^{-1}$ data based on a flat hectare could be divided by the appropriate leaf area index for a more realistic rate of fungus application. Many field studies and commercial labels report a rate of $1.0 \times 10^{11}$ to $1.0 \times 10^{14}$ spores $\mathrm{ha}^{-1}$ which places our $\mathrm{LC}_{50}$ data within the range of field application recommendations.

In our study design we aimed to determine infectivity and virulence of the individual strains of fungi included in the commercialized products, independent of any influence of the formulations. Wraight et al. (2016) showed specific preparations of entomopathogenic fungi in an emulsifiable-oil formulation to be more efficient than both spores alone and wettable powder formulations. This suggests that spores in conjunction with their formulation components may possess greater field efficacy than the spores by themselves.

The primary acquisition testing that has been completed for this screening process has provided insight into the suitability of certain commercial entomopathogenic fungi for the management of $D$. citri populations in the USA. The next stage is field evaluation.
Acknowledgements We would like to thank Mayra Reyes and Clarissa De Leon for their technical assistance in performing some of the experiments. Mention of trade names or commercial products in this publication is solely for the purpose of providing specific information and does not imply recommendation or endorsement by the US Department of Agriculture. Opinions expressed are the author's, based on their expertise, and not necessarily official opinions of the US Department of Agriculture. This material was made possible, in part, by a Cooperative Agreement from the US Department of Agriculture's Animal and Plant Health Inspection Service (APHIS). It may not necessarily express APHIS' views. This research was financially supported by the USDA APHIS Citrus Health Response Program.

Author contributions Not Applicable.

Funding USDA APHIS Citrus Health Response Program.

Availability of data and material Not Applicable.

Code availability Not Applicable.

Open Access This article is licensed under a Creative Commons Attribution 4.0 International License, which permits use, sharing, adaptation, distribution and reproduction in any medium or format, as long as you give appropriate credit to the original author(s) and the source, provide a link to the Creative Commons licence, and indicate if changes were made. The images or other third party material in this article are included in the article's Creative Commons licence, unless indicated otherwise in a credit line to the material. If material is not included in the article's Creative Commons licence and your intended use is not permitted by statutory regulation or exceeds the permitted use, you will need to obtain permission directly from the copyright holder. To view a copy of this licence, visit http://creativecommons.org/licenses/by/4.0/.

\section{References}

Al Mazra'awi MS, Shipp JL, Broadbent AB, Kevan PG (2006) Dissemination of Beauveria bassiana by honeybees (Hymenoptera: Apidae) for control of tarnished plant bug (Hemiptera: Miridae) on canola. Environ Entomol 35(6):1569-1577

Avery PB, Wekesa VW, Hunter WB, Hall DG, McKenzie CL, Osborne LS, Rogers ME (2011) Effects of the fungus Isaria fumosorosea (Hypocreales: Cordycipitaceae) on reduced feeding and mortality of the Asian citrus psyllid, Diaphorina citri (Hemiptera: Psyllidae). Biocontrol Sci Technol 21(9):1065-1078

Bayles BR, Thomas SM, Simmons GS, Grafton-Cardwell EE, Daugherty MP (2017) Spatiotemporal dynamics of the Southern California Asian citrus psyllid (Diaphorina citri) invasion. PLoS ONE 12(3):e0173226

Bové JM (2006) Huanglongbing: a destructive, newlyemerging, century-old disease of citrus. J Plant Pathol 88(1):7-37 
Campbell RK, Anderson TE, Semel M, Roberts DW (1985) Management of the Colorado potato beetle using the entomogenous fungus Beauveria bassiana. Am Potato J 62(1):29-37

Chow A, Dunlap CA, Jackson MA, Avery PB, Patt JM, Sétamou M (2018) Field efficacy of autodissemination and foliar sprays of an entomopathogenic fungus, Isaria fumosorosea (Hypocreales: Cordycipitaceae), for control of Asian citrus psyllid, Diaphorina citri (Hemiptera: Liviidae), on residential citrus. J Econ Entomol 111(5):2089-2100

Chow A, Czokajlo D, Patt JM, Sétamou M (2019) Development and field validation of a beta-cyfluthrin-based 'attract-and-kill' device for suppression of Asian citrus psyllid (Hemiptera: Liviidae) on residential citrus. J Econ Entomol 112(6):2824-2832

Cohen S, Moreshet S, LeGuillou L, Simon JC, Cohen M (1997) Response of citrus trees to modified radiation regime under semi-arid conditions. J Exp Bot 48(306):35-44

Faria M, Wraight SP (2001) Biological control of Bemisia tabaci with fungi. J Crop Prot 20(9):767-778

Flores D, Ciomperlik M (2017) Biological control using the ectoparasitoid, Tamarixia radiata, against the Asian citrus psyllid, Diaphorina citri, in the Lower Rio Grande Valley of Texas. Southwest Entomol 42(1):49-59

Gange AC, Koricheva J, Currie AF, Jaber LR, Vidal S (2019) Meta-analysis of the role of entomopathogenic and unspecialized fungal endophytes as plant bodyguards. New Phytol 223(4):2002-2010

Grafton-Cardwell EE (2006) Asian citrus psyllid. UCANR Publications. http://ipm.ucanr.edu/PMG/PESTNOTES/ pn74155.html

Grafton-Cardwell EE, Stelinski LL, Stansly PA (2013) Biology and management of Asian citrus psyllid, vector of the huanglongbing pathogens. Annu Rev Entomol 58:413-432

Hall DG, Richardson ML, Ammar ED, Halbert SE (2013) Asian citrus psyllid, Diaphorina citri, vector of citrus huanglongbing disease. Entomol Exp Appl 146(2):207-223

Hamdi F, Fargues J, Ridray G, Jeannequin B, Bonato O (2011) Compatibility among entomopathogenic Hyphocreales and two beneficial insects used to control Trialeurodes vaporariorum (Hemiptera: Aleurodidae) in Mediterranean greenhouses. J Invertebr Pathol 108(1):22-29

Jaronski ST, Jackson MA (2012) Mass production of entomopathogenic Hypocreales. In: Lacey LA (ed) Manual of techniques in invertebrate pathology, 2nd edn. Academic Press, New York

Kapongo JP, Shipp L, Kevan P, Broadbent B (2008) Optimal concentration of Beauveria bassiana vectored by bumble bees in relation to pest and bee mortality in greenhouse tomato and sweet pepper. BioControl 53(5):797-812

Khashaveh A, Chelav HS (2013) Laboratory bioassay of Iranian isolates of entomopathogenic fungus Metarhizium anisopliae (Metsch.) Sorokin (Ascomycota: Hypocreales) against two species of storage pest. Agric Conspec Sci 78(1):35-40

Klinger E, Groden E, Drummond F (2006) Beauveria bassiana horizontal infection between cadavers and adults of the
Colorado potato beetle, Leptinotarsa decemlineata (Say). Environ Entomol 35(4):992-1000

Kruse A, Fattah-Hosseini S, Saha S, Johnson R, Warwick E, Sturgeon K, Heck MC (2017) Combining 'omics and microscopy to visualize interactions between the Asian citrus psyllid vector and the Huanglongbing pathogen Candidatus Liberibacter asiaticus in the insect gut. PLoS ONE 12(6):e0179531

Kumar V, Avery PB, Ahmed J, Cave RD, McKenzie CL, Osborne LS (2017) Compatibility and efficacy of Isaria fumosorosea with horticultural oils for mitigation of the Asian citrus psyllid, Diaphorina citri (Hemiptera: Liviidae). Insects 8(4):119

Litchfield JT, Wilcoxon F (1949) A simplified method of evaluating dose-effect experiments. J Pharmacol Exp Ther 96(2):99-113

Meyling NV, Pell JK (2006) Detection and avoidance of an entomopathogenic fungus by a generalist insect predator. Ecol Entomol 31(2):162-171

Morgan KT, Barkataky S, Kadyampakeni D, Ebel R, Roka F (2014) Effects of short-term drought stress and mechanical harvesting on sweet orange tree health, water uptake, and yield. HortScience 49(6):835-842

Osborne LS, Landa Z (1992) Biological control of whiteflies with entomopathogenic fungi. Fla Entomol 75(4):456-471

Pelz-Stelinski KS, Brlansky RH, Ebert TA, Rogers ME (2010) Transmission parameters for Candidatus liberibacter asiaticus by Asian citrus psyllid (Hemiptera: Psyllidae). J Econ Entomol 103(5):1531-1541

Roy HE, Pell JK (2000) Interactions between entomopathogenic fungi and other natural enemies: implications for biological control. Biocontrol Sci Technol 10(6):737-752

Schneider CA, Rasband WS, Eliceiri KW (2012) NIH Image to ImageJ: 25 years of image analysis. Nat Methods 9(7):671-675

Sétamou M, Alabi OJ, Kunta M, Dale J, da Graça JV (2020) Distribution of Candidatus Liberibacter asiaticus in citrus and the Asian citrus psyllid in Texas over a decade. Plant Dis 104(4):1118-1126

Spence E, Hesketh H, Svendsen C, Chandler D, Martin G, Berry SD, Edgington S (2019) Screening potential entomopathogenic fungi for the control of the greenhouse whitefly (Trialeurodes vaporariorum). Unpublished

Tasin M, Knudsen GK, Pertot I (2012) Smelling a diseased host: grapevine moth responses to healthy and fungusinfected grapes. Anim Behav 83(2):555-562

Wraight SP, Carruthers RI (1999) Production, delivery, and use of mycoinsecticides for control of insect pests on field crops. Biopesticides: use and delivery. Humana Press, Totowa, pp 233-269

Wraight SP, Carruthers RI, Bradley CA, Jaronski ST, Lacey LA, Wood P, Galaini-Wraight S (1998) Pathogenicity of the entomopathogenic fungi Paecilomyces spp. and Beauveria bassiana against the silverleaf whitefly, Bemisia argentifolii. J Invertebr Pathol 71(3):217-226

Wraight SP, Carruthers RI, Jaronski ST, Bradley CA, Garza CJ, Galaini-Wraight S (2000) Evaluation of the entomopathogenic fungi Beauveria bassiana and Paecilomyces fumosoroseus for microbial control of the silverleaf whitefly, Bemisia Argentifolii. Biol Control 17(3):203-217 
Wraight SP, Ramos ME, Avery PB, Jaronski ST, Vandenberg JD (2010) Comparative virulence of Beauveria bassiana isolates against lepidopteran pests of vegetable crops. J Invertebr Pathol 103(3):186-199

Wraight SP, Filotas MJ, Sanderson JP (2016) Comparative efficacy of emulsifiable-oil, wettable-powder, and unformulated-powder preparations of Beauveria bassiana against the melon aphid Aphis gossypii. Biocontrol Sci Technol 26(7):894-914 